\title{
Cd versus web: algunos ejemplos en biomedicina
}

\section{Por Judith Prieto Ayuda y Laura Casado Castells}

Resumen: A lo largo del último cuarto de siglo las nuevas tecnologías de la información han traído al mundo de la documentación una gran herramienta de almacenamiento, acceso, consulta y recuperación de la información: las bases de datos. Ahora, en pleno siglo XXI, aparece un dilema: ¿bases de datos en cd o vía web? Este artículo intenta identificar algunos de los

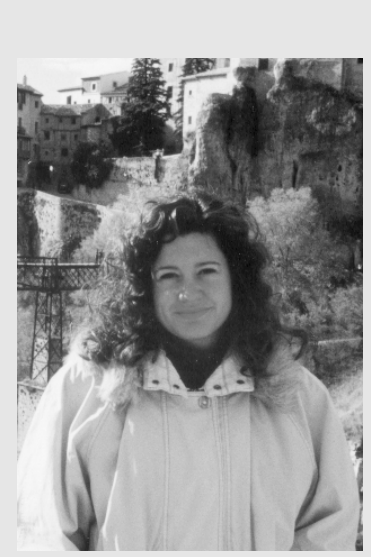
pros y contras, así como mencionar algunos casos en biomedicina.

Palabras clave: Bases de datos, Cd-rom, Web, Biomedicina.

Title: Cd-rom versus web: some examples in the biomedical sciences

Abstract: Over the past quarter of a century, evolving information technologies have brought to the world of document control a basic tool for storing, accessing and retrieving information: the database.

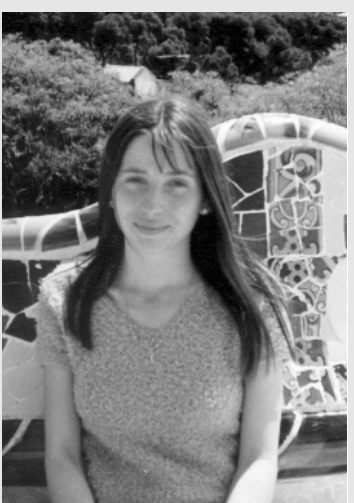

Judith Prieto Ayuda At present, in the 21st century, a dilemma appears: databases on cd-rom or on websites? This article attempts to identify the pros and cons, and mentions some examples from the area of biomedical science.

Laura Casado Castells Keywords: Databases, Cd-rom, Website, Biomedicine. 


\title{
BASES DE DATOS
} REFERENCIALES

\author{
$\checkmark$ Artículos publicados en todas las revistas \\ españolas a partir de 1970 \\ $\checkmark$ Fondos de todas las bibliotecas del Consejo \\ Superior de Investigaciones Cientificas \\ $\checkmark$ Proyectos y líneas de Investigación
}

\section{OFERTA ESPECIAL}

CONSULTA GRATUITA

DURANTE TODO EL MES DE NOVIEMBRE 
EN LA FECHA DE PUBLICACIÓN de este artículo, las bibliotecas y centros de documentación comenzarán a trabajar en sus informes otoñales sobre qué bases de datos, revistas y libros electrónicos han decidido suscribir para el próximo año. A factores como la temática $y$ especialización de cada producto o los intereses de los usuarios, habrá que sumar muchos otros que nos llevarán a la elección definitiva.

En este texto nos centramos en un nuevo ámbito, surgido hace escasos años, pero con una importancia en aumento. Se trata del dilema que viven muchas instituciones a la hora de elegir entre contratar productos accesibles a través de internet o hacerlo en su versión cd-rom, tanto para ordenadores monopuesto como para instalarlos en una red interna. Ambos sistemas presentan ventajas e inconvenientes que inclinarán la balanza hacia una u otra elección dependiendo de las características de la organización en concreto. El objetivo será siempre encontrar el mejor modelo para el usuario, el cual, a priori, no tiene predilección por ninguna opción y lo único que le interesa es acceder, consultar y recuperar la información que necesita en el momento preciso.

\section{Ventajas e inconvenientes de ambos sistemas}

Partiendo de la base de que los contenidos son idénticos, cabe resaltar que los productos en cd-rom tienen siempre una actualización más tardía que los alojados en el servidor del proveedor.

Este es el caso de Current contents (figura 1), base de datos producida por el Institute for Scientific Information (ISI), que corresponde a la versión impresa de las series que llevan el mismo nombre. Estas

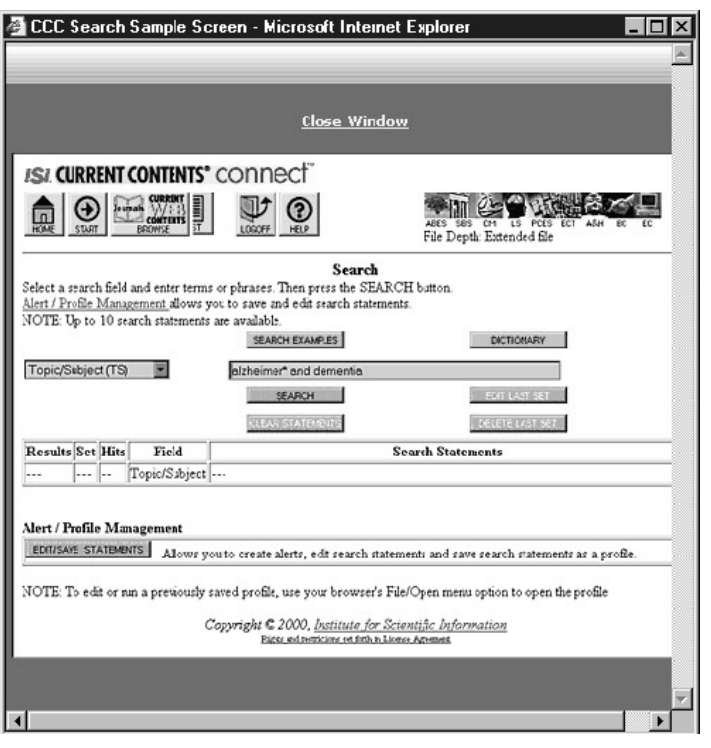

Figura 1

series son boletines de sumarios de periodicidad semanal donde se indizan más de 1.200 revistas de todo el mundo especializadas en biología, bioquímica, microbiología e investigación biomédica. En su versión web (Web of science) la actualización es inmediata y el acceso a la información corresponde realmente a la semana en curso. En cambio, en cd-rom los procesos de almacenaje de la información, envío e instalación hacen que el acceso se haga con un retraso de unas 2 ó 3 semanas.

http://www.isinet.com/isi/product s/cc/ccconnect/cccsitewide/index. html http://www.isinet.com

«Partiendo de la base de que los contenidos son idénticos, cabe resaltar que los productos en cd-rom tienen siempre una actualización más tardía que los alojados en el servidor del proveedor»

Otro ejemplo lo encontramos en UpToDate (figura 2), base de datos referencial, especialmente dirigida a internistas de hospital, que incluye el equivalente a 40.000 páginas de texto original y revisado, más de 8.000 gráficos, 100.000 abstracts extraídos de Medline y una base de datos sobre medicamentos. Un equipo de expertos se encarga de revisar el contenido de unas 200 revistas escogiendo la información más relevante para incorporarla con actualización cuatrimestral. En la versión online la base de datos reside en el servidor del editor, con lo que el centro usuario no debe preocuparse ni del mantenimiento ni de las actualizaciones, que se hacen de manera automática.

http://www.uptodate.com/

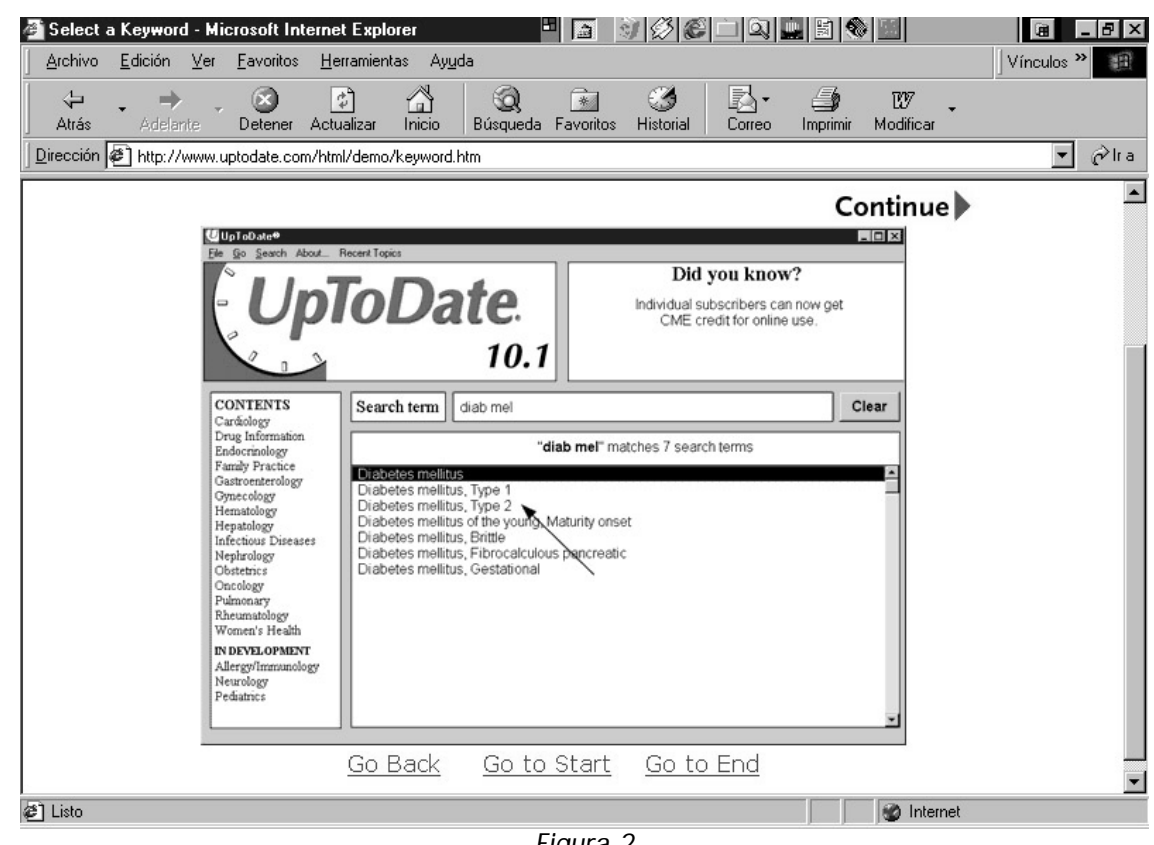

Figura 2 


\section{Complete su colección de IWE/EPI}

Deseo recibir las siguientes publicaciones:

_ _-_ ejemplares de la Bibliografía IWE/EPI 1992-2001 por correo postal impresos en papel (gratis).

la Bibliografía IWE/EPI 1992-2001 por correo electrónico en formato PDF (gratis).

$\square$ _-- álbumes de los 2 cd-roms con el texto íntegro de los primeros 100 números de la revista a 30 euros cada álbum (1 álbum es gratis para los suscriptores).

$\square$ _-- ejemplares de los siguientes números atrasados de la revista (por favor, envíenme presupuesto).

N ombre:

Institución:

D pto.: ............................................................. IF institucional:

Dirección:

Código postal:

Ciudad:

País:

Teléfono:

Fax:

Correo-e:

Envíe este boletín por correo postal, fax o correo electrónico, a esta dirección:

\section{Swets Blackwell \\ Caspe, 46 \\ 08010 Barcelona \\ España}

Tel.: +34-932 701 144; fax: 932701145

mnzang@es.swetsblackwell.com

Un tema de especial interés es la calidad de las conexiones a internet. Considerada una de las principales desventajas de los productos online, su importancia se va relativizando a medida que mejora el acceso a la Red y tendrá mucho que ver con el servidor de acceso del centro en cuestión. Si se caracteriza por su rapidez y seguridad, la consulta a los productos no representará ningún problema. Si por el contrario la red está sobrecargada, con un estrecho ancho de banda y frecuentes cortes en las transmisiones, la posibilidad de poder acceder a los productos contratados se convierte en remota. Cabe destacar que las infraestructuras requeridas por los productos docu- mentales pueden ser aprovechadas por la organización para proporcionar otros servicios, tanto en sistemas internos, como de acceso remoto a recursos de interés.

\section{«Las infraestructuras requeridas por los productos documen- tales pueden ser aprovechadas por la institución para pro- porcionar otros servi- cios»}

Es evidente que la salida a internet representa a priori menos seguridad que la consulta de un cdrom. No obstante, los productos van actualizando a diario sus niveles de seguridad garantizando tanto la protección de datos de los clientes como las aplicaciones. Las opciones de autentificación más comunes son a través de la dirección ip del centro (nombre/número de dominio registrado por éste en internet), o bien por nombre de usuario y password si aquella es compartida con otros centros.

A continuación vamos a comentar un tema que resulta vital para el documentalista, preocupado siempre por garantizar el acceso de sus usuarios a la información de forma permanente. Hay que tener presente que se permite el acceso a los productos online sólo mientras 


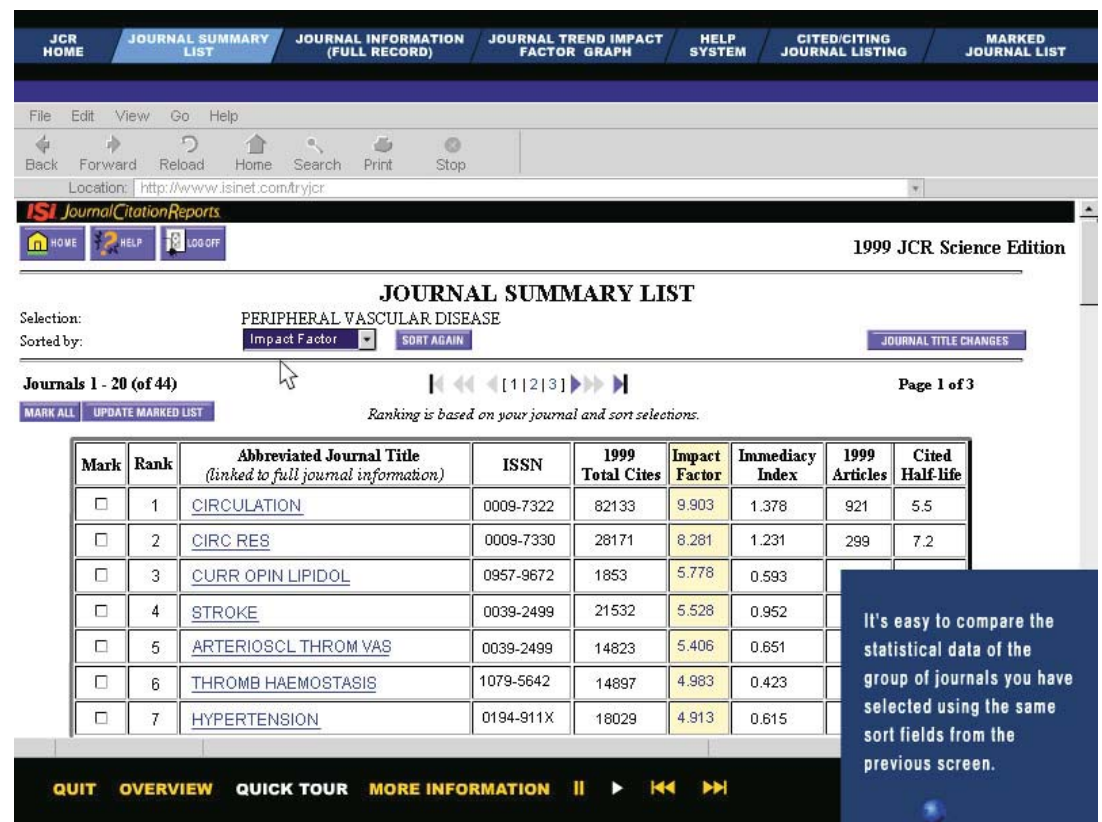

Figura 3

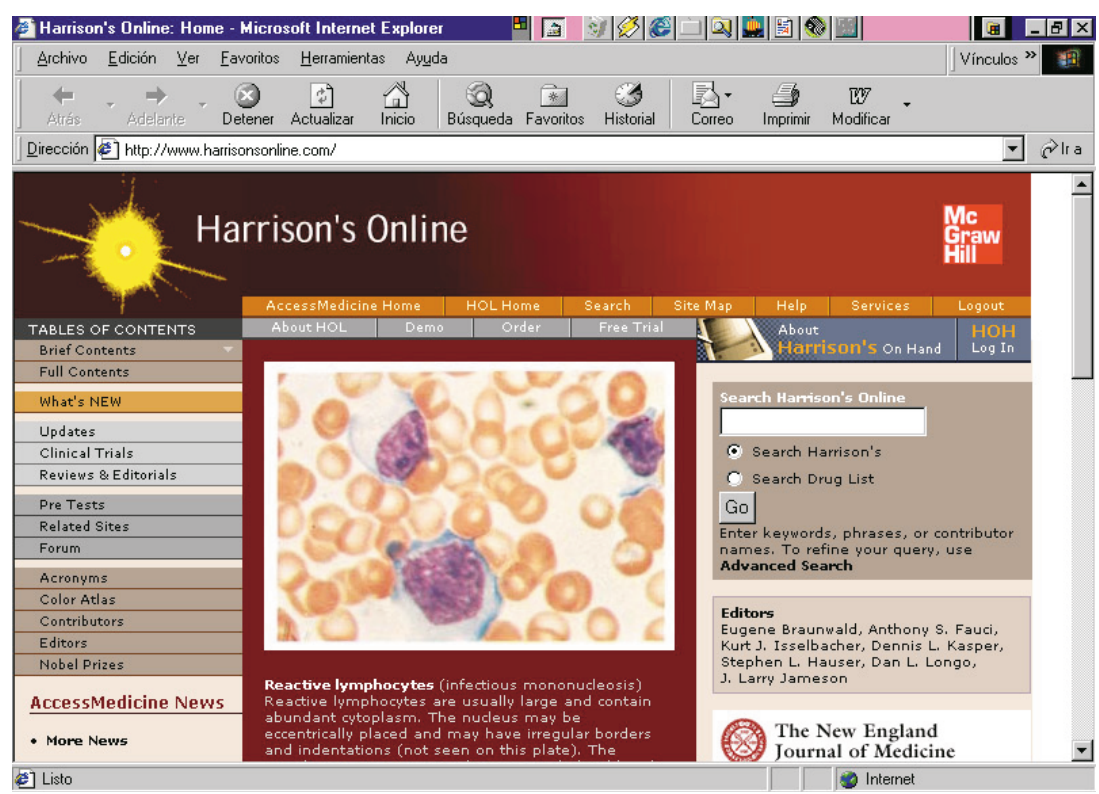

Figura 4

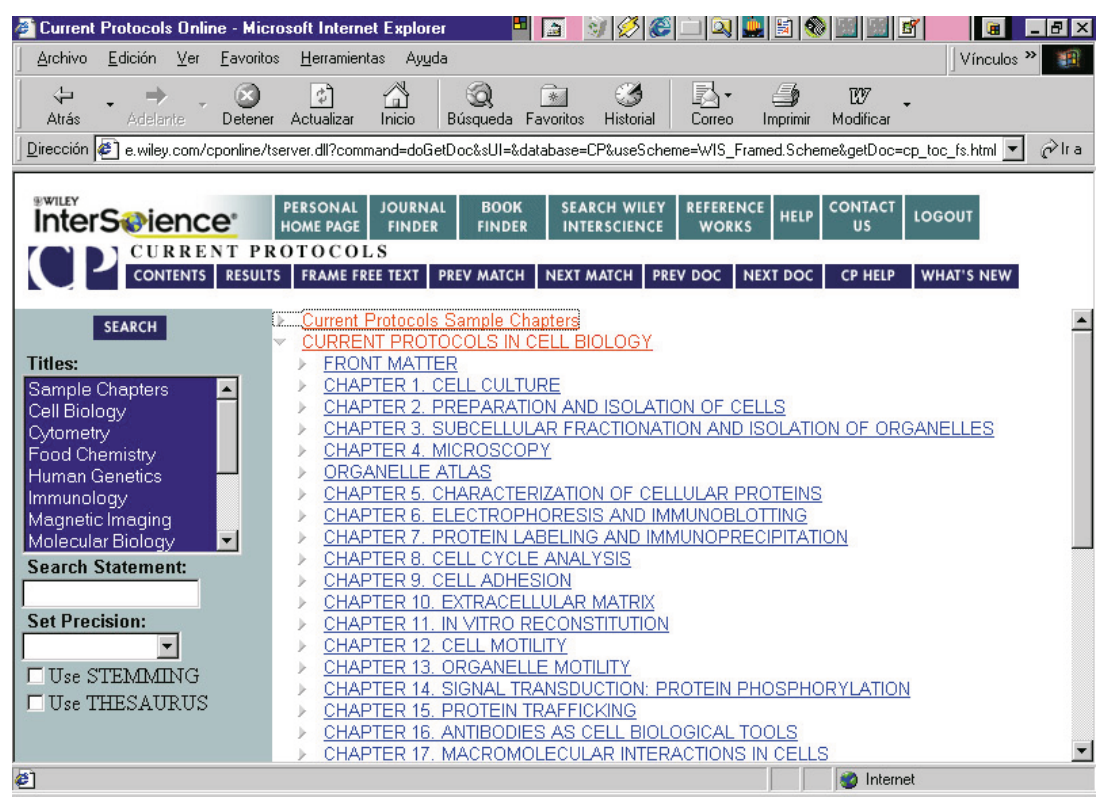

Figura 5 dura el período de vigencia de la suscripción. Una vez ha finalizado, el centro de documentación pierde todo acceso a los datos que hasta la fecha había podido consultar. En cambio, si la suscripción es en cdrom, seguirán en su poder $\mathrm{y}$, por tanto, podrán continuar siendo utilizados.

Un caso muy significativo es el del Journal citation reports (figura 3), base de datos producida por el $I S I$. El JCR es un recurso de información esencial y único en el mercado para la evaluación de revistas científicas incluyendo, en su Science edition, la referencia de más de 5.000 publicaciones en las áreas de ciencia y tecnología. Es una herramienta básica para la redacción de los curriculums vitae, siendo el factor de impacto de las publicaciones uno de los baremos más internacionalmente reconocidos para cuantificar la producción científica de un grupo de investigación. Es precisamente en la elaboración de los curriculums donde es necesario disponer no sólo de la última edición del $J C R$, si no de las anteriores, dado que cada publicación se evalúa con el índice de impacto correspondiente al año anterior al de su publicación.

\section{http://www.isinet.com/isi/products/ citation/jcr/index.html}

No se puede olvidar tampoco un aspecto básico a la hora de determinar la política de adquisiciones y el marketing de nuestros productos: las estadísticas. Las aplicaciones online acostumbran a ofrecer en sus paquetes de acceso estadísticas de uso, de forma que podemos conocer el número de usuarios y sus pautas de consulta. En el caso del cd-rom, normalmente es el propio centro el que debe crear esta opción.

Los servicios de valor añadido serían realmente el factor de diferencia más visible entre ambos soportes. Frecuentemente, la versión 


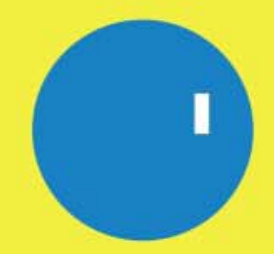

O V I D

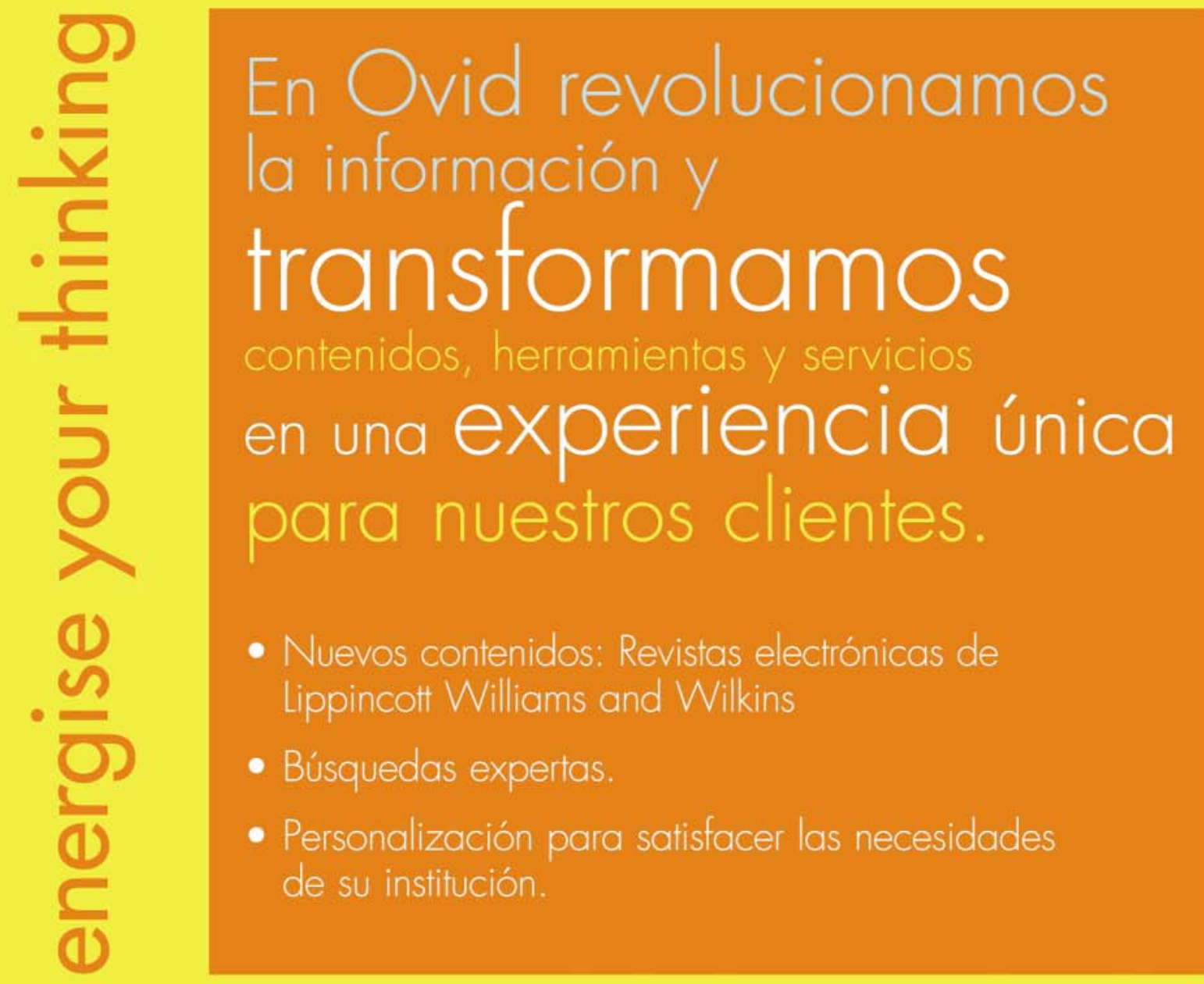

Madrid tél 914186275 Barcelona tél 938106563 spain@ovid.com 
online de un producto ofrece más prestaciones, como puede ser la consulta simultánea de bases de datos, mayores herramientas de búsqueda y de navegación, etc.

Un ejemplo lo encontramos en Harrison's online, versión electrónica del libro Harrison's principles of internal medicine, que incluye enlaces a otras publicaciones de sus autores indizadas en Medline (figura 4). Así mismo, ofrece un apartado de enlaces de interés estructurado en áreas temáticas.

\section{http://www.harrisonsonline.com/}

Otro ejemplo, en este caso una combinación de ambos (cd-rom y web), sería el libro Mandell, Douglas and Bennett's principles and practice of infectious diseases on cd-rom, versión electrónica de la obra del mismo nombre, que versa sobre microbiología, inmunología y tratamiento de agentes infecciosos. Desde el cd se accede directamente a PubMed mediante enlaces que van desde las referencias bibliográficas al abstract.

\section{«Las aplicaciones on- line acostumbran a ofrecer en sus pa- quetes de acceso es- tadísticas de uso, de forma que podemos conocer el número de usuarios y sus pau- tas de consulta»}

Por último, citaremos la siempre básica cuestión presupuestaria. En este sentido, nos encontramos una vez más sin reglas fijas. En algunas ocasiones no se incrementa el precio de la suscripción online; en otras el aumento es considerable, llegando a triplicar el precio de la versión en cd. Nuevamente, será necesario el estudio de la oferta de cada producto en cuestión antes de tomar una decisión en un sentido $u$ otro. Algunos ejemplos de bases de datos, ya mencionadas anterior- mente, serían el Web of science de ISI (que incluye las bases de datos Current contents y Journal citation reports) y UpToDate. Además nos encontramos con el Current protocols (figura 5), publicación dirigida a los laboratorios de investigación y formada por una serie de manuales que tratan sobre biología molecular, inmunología, genética, proteínas, citometría, neurociencias, farmacología, biología celular, toxicología y química del ácido nucleico.

http://www.wiley.co.uk/cp/cppub.htm

\section{Conclusión}

La máxima que debe regir al documentalista a la hora de decantarse por un sistema u otro debe ser establecida teniendo en cuenta las características de su centro y de los productos que desea contratar. La experiencia ajena no servirá de ayuda, dado que cada organismo presenta su propia casuística tanto a nivel de infraestructura tecnológica y de comunicaciones como de productos requeridos. Finalmente, temas como la preservación y acceso de los documentos son aún retos por resolver en ambas opciones.

\section{Bibliografía}

Baiget, Tomàs. "Hosts y bases de datos en la era web". En: El profesional de la información, 2001, marzo, v. 10, n. 3, pp. 19-20.

Alòs-Moner, Adela d'; Ferran, Núria. "Del elefante a internet: breve historia de las bases de datos y tendencias de futuro". En: El profesional de la información, 2001, marzo, v. 10, n. 3, pp. 22-26.

Alvarado Gómez, Francisco; Izquierdo Moya, Pilar. "Consultas bibliográficas automatizadas del cd-rom a internet". En: Actas de las VIII Jornadas de información y documentación en ciencias de la salud, 2001, pp. 184-189.

Rodríguez del Castillo Martín, Mercedes. "Tendencias que presentan las bibliotecas de ciencias de la salud". En: El profesional de la información, 2000, diciembre, v. 9, n. 12, pp. 4-12.

Gethin, Peter. "Automatización de bibliotecas”. En: El profesional de la información, 2001 , noviembre, v. 10 , n. 11 , pp. 26-31.

Judith Prieto Ayuda, Agencia para la Gestión del Conocimiento y la Do- cencia, Hospital Universitario Vall d'Hebron, Paseo de la Vall d'Hebron, 119-129, 08035 Barcelona.

Tel.: +34-934 894 012;

fax: 934-894014

judith@cs.vhebron.es

Laura Casado Castells, Dirección de Investigación, Hospital Universitario Vall d'Hebron, Paseo de la Vall d'Hebron, 119-129, 08035 Barcelona.

Tel.: +34-934894 011;

fax: 934-894 102

laura@cs.vhebron.es 